\title{
Multiple subtraction using an expanded multichannel matching filter
}

\author{
Yanghua Wang*
}

\section{ABSTRACT}

An expanded multichannel matching (EMCM) filter is proposed for the adaptive subtraction in seismic multiple attenuation. For a normal multichannel matching filter where an original seismic trace is matched by a group of multiple-model traces, the lateral coherency of adjacent traces is likely to be exploited to discriminate the overlapped multiple and primary reflections. In the proposed EMCM filter, a seismic trace is matched by not only a group of the ordinary multiple-model traces but also their adjoints generated mathematically. The adjoints of a multiple-model trace include its first derivative, its Hilbert transform, and the derivative of the Hilbert transform. The convolutional coefficients associated with the normal multichannel filter can be represented as a 2D operator in the time-space domain. This 2D operator is expanded with an additional spatial dimension in the EMCM filter to improve the robustness of the adaptive subtraction. The multiple-model traces are generated using moveout equations to afford efficiency in the multiple attenuation application.

\section{INTRODUCTION}

There are various techniques for attenuating the water-layer peg-leg multiples in marine seismic data, though their effectiveness depends on the water depth. For predictive deconvolution, which uses the periodicity in the behavior of multiples, a rule of thumb is that the window length is six to ten times as long as the maximum multiple period. In the deep-water case where the length of the available record isn't long enough compared to the reverberation period, poor autocorrelation estimates result in the predictive deconvolution not working. The group of techniques such as $f-k$ and $\tau$ - $p$ filtering, exploiting the velocity difference between primaries and multiples, may not work either because in the deep-water case the differential moveout between the multiple and the primary is very small.
The wave-equation-based methods, which are two-step procedures consisting of multiple modeling and subtraction, can be used in the general situation (Berryhill and Kim, 1986; Wiggins, 1988; Verschuur et al., 1992; Berkhout and Verschuur, 1997; Verschuur and Berkhout, 1997). But a relatively long computational time required for the multiple prediction is a factor of consideration and, most importantly, the effectiveness of the method depends upon the adaptive subtraction of the multiple model and the original data traces. In this paper, to afford efficiency, moveout equations are used to predict the water-layer multiples generated by the primary energy being trapped in the water column (Appendix A). The emphasis, however, is on the importance of the second step, the adaptive subtraction.

Multiple subtraction is posed as a least-squares minimization problem that minimizes the energy difference between the original data traces and the multiple-model traces. It implemented as multichannel matched filtering, exploiting the lateral coherency of adjacent traces as a constraint to discriminate primaries from overlapping multiples. When a normal multichannel matching filter matches a data trace to a group of model traces, the associated convolution coefficients can be represented in a group as a $2 \mathrm{D}$ operator in the timespace domain. In this paper, I present a so-called expanded multichannel matching filter, which expands the $2 \mathrm{D}$ operator with an additional dimension. It matches an input trace to a group of multiple-model traces and their adjoints generated mathematically, so as to make the adaptive subtraction robust.

The adjoints of a multiple-model trace include its first derivative, its Hilbert transform, and the derivative of the Hilbert transform. These adjoint traces were used by Monk (1993) in constrained cross-equalization which, using the amplitude scalar operator, considered the constant phase rotation of the wavelet and the constant time shift. In contrast, the expanded multichannel matched filtering, using convolutional operators, recognizes that both the amplitude and phase changes between the data trace and the multiple-model traces are frequency dependent, and the time shift of the multiple wavelets is time variant as well.

Manuscript received by the Editor October 18, 2001; revised manuscript received July 29, 2002.

*Robertson Research International Ltd., Horizon House, Azalea Drive, Swanley, Kent BR8 8JR, United Kingdom. E-mail: yanghua@geo. robresint.co.uk.

(C) 2003 Society of Exploration Geophysicists. All rights reserved. 


\section{MULTICHANNEL MATCHING FILTERS}

Once the multiple model traces are predicted based on the original data set or a predetermined subsurface model, the subtraction of modeled multiples from the original traces is carried out by a single or multichannel matched filtering within a sliding window. The matching filter may be represented as

$$
p(t)=y(t)-\sum_{j=1}^{N} f_{j}(t) * m_{j}(t),
$$

where $y(t)$ is a raw data trace, $m_{j}(t)$ are multiple model traces, $N$ is the number of channels involved in matching, $f_{j}(t)$ are the operators for adapting the group of $N$ traces $m_{j}(t)$ to the desired output $y(t), *$ indicates convolution, and $p(t)$ is the residual. For multiple subtraction, the residual $p(t)$ is the multiple suppressed result, whereas $f_{j}(t)$ are referred to as the subtraction operators.

The matching filter uses the least-squares criterion to design operators $f_{j}(t)$ so as to minimize the power in a selected window for the desired output $y(t)$ (Treitel, 1970). The design error of the shaping filter, which shapes the single or a group of multiple-model traces into the original data trace, is considered as the primary response. The convolutional operators $f_{j}(t)$ are solved recursively, following Wiggins and Robinson (1965).

If using a single channel convolutional filter without constraints, it is possible to match anything to anything. The simplest constraint is to use an amplitude scalar operator. If we assume that the multiple-model trace $m(t)$ only have a scalar difference with the corresponding multiple in the input data trace $y(t)$, we could use an amplitude scalar

$$
w(t)=\frac{\sum_{\tau=-T / 2}^{T / 2} y(t+\tau) m(t+\tau)}{\sum_{\tau=-T / 2}^{T / 2} m^{2}(t+\tau)}
$$

to subtract the multiple model from the input trace:

$$
p(t)=y(t)-w(t) m(t)
$$

However, if the primary and multiple are nonorthogonal in the scalar estimation window $T$, it is almost impossible to obtain an exact subtraction scalar (Zhou, 1993).

The constrained cross-equalization method (Monk, 1993) is the extension of the above amplitude scalar operator. It considered also the phase rotation of the wavelet and the time shift. It adapted the multiple model trace using a weighted sum of four traces which could each be derived from the initial multiplemodel trace. The constrained cross-equalization filter may be expressed as

$$
\begin{aligned}
p(t)= & y(t)-\left[w_{1} m(t)+w_{2} \dot{m}(t)\right. \\
& \left.+w_{3} m^{H}(t)+w_{4} \dot{m}^{H}(t)\right],
\end{aligned}
$$

where $m^{H}(t)$ is the Hilbert transform of $m(t), \dot{m}(t)$ and $\dot{m}^{H}(t)$ are the derivatives of $m(t)$ and $m^{H}(t)$, respectively, and $w_{i}$ are weighting coefficients. This method consists of the following three fundamental assumptions (Appendix B):

1) The amplitude difference between $m(t)$ and $y(t)$ is a scalar.
2) The phase difference is a constant rotation.

3) The time difference is also a constant.

Although the constant amplitude scale and constant time shift may serve as constraints for the matching, the assumption of simple constant phase rotation can not adequately describe the waveform change between primary and multiple reflections.

In reality, the amplitude difference and the phase change both are frequency dependent, and the time shift is also time variant. Therefore, a convolutional operation seems quite practical:

$$
\begin{aligned}
p(t)= & y(t)-\left[f_{1}(t) * m(t)+f_{2}(t) * \dot{m}(t)\right. \\
& \left.+f_{3}(t) * m^{H}(t)+f_{4}(t) * \dot{m}^{H}(t)\right]
\end{aligned}
$$

where $f_{i}(t)$ are convolutional operators and $*$ indicates convolution. The derivation of this convolution formula is given in Appendix B. The implementation of equation (5) is the same as the multichannel matching filter (1), but three channels other than $m(t)$ are originated from the single channel $m(t)$. It is thus referred to as the pseudomultichannel matching filter.

Combining the normal and pseudomultichannel matching filters (1) and (5), we have an expanded multichannel matching filter defined as

$$
\begin{aligned}
p(t)= & y(t)-\sum_{j=1}^{N}\left[f_{1, j}(t) * m_{j}(t)+f_{2, j}(t) * \dot{m}_{j}(t)\right. \\
& \left.+f_{3, j}(t) * m_{j}^{H}(t)+f_{4, j}(t) * \dot{m}_{j}^{H}(t)\right],
\end{aligned}
$$

where $N$ is the number of traces used in the preceding normal multichannel matching filter (1).

In the normal multichannel matching filter, trace $y(t)$ is matched by a $N \times N_{t}$ section, where $N$ is the number of traces and $N_{t}$ is the number of time samples in the matching window. In the expanded version (6), trace $y(t)$ is matched by a data cube of $4 \times N \times N_{t}$, in which three out of four sections are adjoint sections mathematically derived from the original data section. It is called the expanded multichannel matching filter, to reflect the expansion in the new physical dimension.

\section{COMPARISON OF FOUR DIFFERENT MATCHING FILTERS}

In summary, we have four different types of adaptive matching filters:

1) The single channel matching filter [equation (1) with $N=1]$.

2) The normal multichannel matching filter [equation (1) with $N>1$ ].

3) The pseudomultichannel matching filter in which the four channels are originated from a single channel [equation (5), or equation (6) with $N=1$ ].

4) The expanded multichannel matching filter, which consists of the original multichannels and the three adjoint traces of each of them [equation (6) with $N>1$ ].

These four matching operations are drawn in Figure 1 schematically.

A comparison of the effectiveness of these different matching filters is shown in Figure 2, where (a) is the original data window where a primary and a multiple reflection overlap; 
(b) is the predicted multiple model, which is the same as the multiple event in the original gather except for a scaling factor; (c) is then the multiple attenuation result obtained from using the single channel matching filter; (d) is the demultiple result using the normal multichannel matching filter; (e) is the demultiple result using the pseudomultichannel matching filter in which the channels are originated from a single trace; and finally, (f) is the result obtained by using the expanded multichannel matching filter. Figure 2 displays the results for a limited time window, although other primary and multiple events are present outside this window.

The single channel filter generally suffers from the problem that it cannot separate the primary energy from the multiple energy if they were overlapped. The result is essentially a "blank out" of all data where the multiple was present. Figure $2 \mathrm{c}$ is a typical example of the results obtained from this type of process, regardless of the method used to generate the multiple model traces. A perfect removal of multiples from the original data trace can be carried out only if the primaries and multiples do not overlap, or if the primaries constitute a totally random time series in the window of interest.

Figures $2 \mathrm{~d}$ and $2 \mathrm{e}$ compare the normal and the pseudomultichannel matching filters, both having effectively four channels in filter designing. The normal multichannel matching filter improves the preceding single channel filtering result by filling the energy residual in the blank-out gap. The pseudomultichannel matching filter in which three out of four channels are originated from the single trace, however, seems more effective than the normal one in terms of preservation of the primary energy. Although both used exactly the same parameters of the

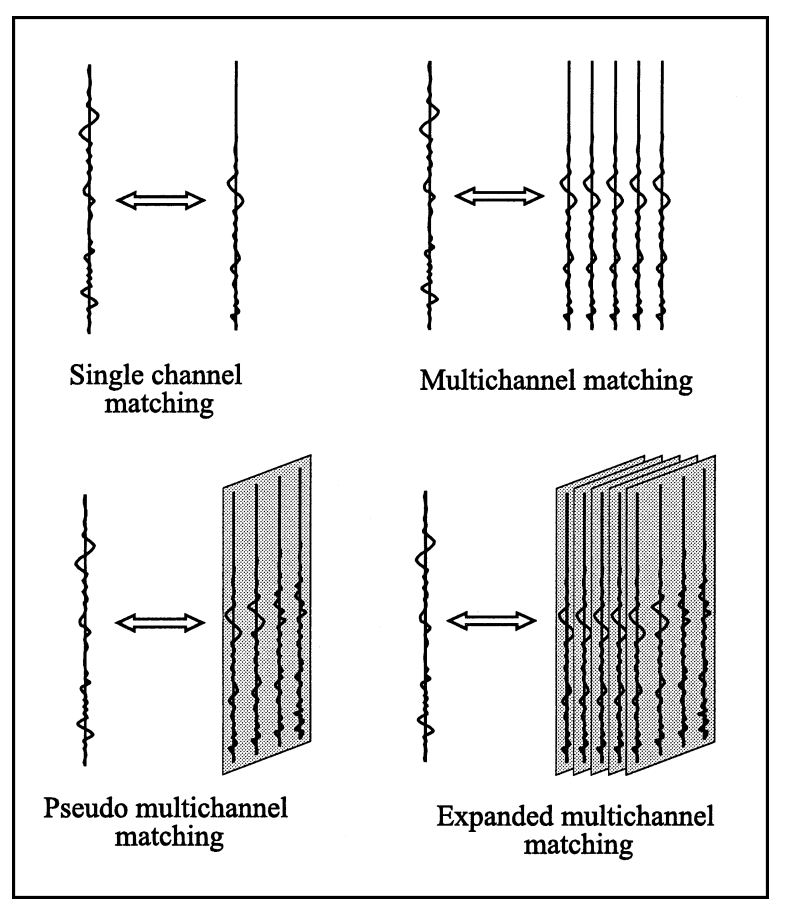

FIG. 1. Four different matching filters: the single channel matching filter, the normal multichannel matching filter, the pseudomultichannel matching filter in which the channels are originated from a single trace, and the expanded multichannel matching filter, which consists of the original multichannels and the three adjoint traces of each of them. window size and the filter length for the sake of fair comparison, Figure $2 \mathrm{e}$ has more multiple energy remaining in overall data window than Figure 2d.

The expanded multichannel matching filter produces the superior multiple attenuation result shown in Figure 2f, compared to the others. In the preceding normal multichannel filter (Figure 2d), we hope that the lateral coherency might be used implicitly as a constraint to discriminate multiples and primaries. In the expanded version here, the lateral coherence is measured not only on the ordinary traces, but also on the Hilbert traces and on the derivatives of the ordinary and Hilbert traces. In the pseudomultichannel matching filter (Figure 2e), we attempt to discriminate multiples and primaries by exploiting information from mathematically derived traces. The combination of these two dimensional discriminations in the expanded multichannel matching filter (Figure 2f) results in the excellent preservation of the continuity of the primary event.

Note that results shown in Figure 2 are subject to the choice of filter parameters and the implementation. A separate filter for each trace is calculated and applied to the individual trace. In the case of single channel filtering, however, we could calculate one single filter for the entire record. The filter can be even degenerated into a single scalar, provided the multiple and primary are orthogonal, following equation (2). In the case of multichannel filtering, however, we cannot apply a single multichannel filter for entire record because of the curvature of events, but need a group of multichannel filters.

Monk's (1993) cross-equalization constrains the changes allowed in the model trace by limiting the number of free parameters to four, as in equation (4). Changing the simple weights to the filter operators will increase the degree of freedom allowed in matching. In the typical example shown in Figure 2, the convolutional filters are constrained to be short, assuming very smooth variations of amplitudes and shifts with frequency. For a fair comparison, the filter length of all filters is set to be two points for either single or multichannel filtering. But they differ in the window size. For single channel filtering, the window size is $400 \mathrm{~ms}$. For the normal multichannel and pseudomultichannel filtering, the window size is $800 \mathrm{~ms}$. For the expanded multichannel filtering, the window size is further doubled to $\geq 1600 \mathrm{~ms}$, though Figure 2 only shows a zoomed-in window between 1200 and $1500 \mathrm{~ms}$.

\section{APPLICATION EXAMPLE}

The expanded multichannel matching filter is interesting on its own and can be plugged into existing wave-equation-based, two-phase multiple attenuation software. In Appendix A, I describe a moveout-equation-based multiple modeling method which can offer great efficiency. Each data sample in the seismic record is assumed to be primary energy. The multiple arrival time is calculated using the moveout equation, as if the wave bounces additionally once or twice within the water layer, and then transform the data sample to the time position of the expected multiple. This method requires only the knowledge of the rms velocity function and the vertical two-way time of the water layer, whereas the water bottom is assumed to be flat locally. The multiple attenuation method, using moveoutequation-based multiple prediction in conjunction with the expanded multichannel matching filter, is referred to as MEMUL. 
The focus point however is to demonstrate that a multiple attenuation technique would work essentially if we have had an appropriate subtraction procedure. As a demonstration, we apply MEMUL to a 2D synthetic data set, Pluto 1.5, which was designed for benchmarking multiple attenuation algorithms and supplied by the SMAART Joint Venture Consortium (Bishop et al., 2001; Stoughton et al., 2001).

Figure $3 \mathrm{a}$ shows an example of input common-midpoint (CMP) gather which is treated as a "source" of primaries to generate the peg-leg multiples in Figure 3b, whereas the firstorder peg-leg multiples in the original gather are mapped to be the second-order ones. The wavelet in the multiple model differs in time, amplitude, and phase from that in the original data gather. These differences in fact challenge the robustness of the matching filter in the subsequent subtraction. Figure $3 \mathrm{c}$ displays the multiple energy removed after the adaptive subtraction, whereas Figure $3 \mathrm{~d}$ is the result of MEMUL with expanded multichannel matching filter.
When applying the matching filter to CMP gathers, NMO correction (with water-layer velocity) may be applied to both the original data gather and the multiple-model gather, and can improve the multichannel matching effect on far-offset traces typically in shallow water and/or long-offset cases.

A stack of the synthetic data set is displayed in Figure 4, which shows clearly the strong multiples with a wide range of dips generated at the water bottom and salt-sediment interfaces. In the figure, the steeply dipping diffractions appear to be aliased just because we plot only every sixth trace in the actual data set. We test the MEMUL method using the expanded multichannel matching filter in the CMP domain and in the common-offset domain.

The CMP-domain MEMUL result is shown in Figure 5, where (a) is the stack section after multiple attenuation and (b) is the energy removed by the demultiple processing. The multiples have been removed effectively in Figure 5a without wiping off the primary energy, although Figure $5 b$, the difference
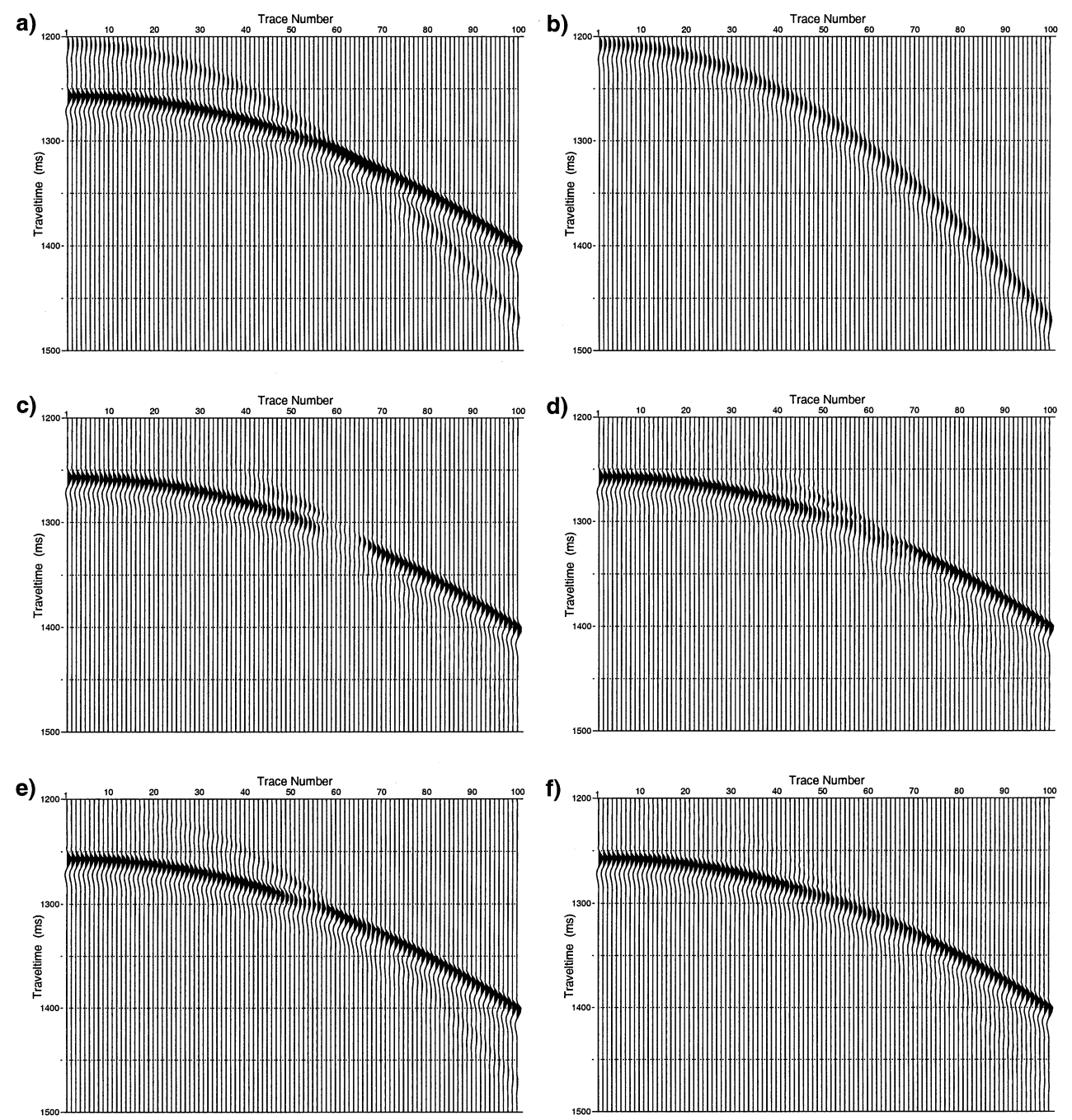

FIG. 2. Comparison of the effectiveness of different matching filters: (a) the original data window where the primary and multiple reflections overlap; (b) predicted multiple model, which is the same as the multiple event in the original gather except for scaling factor; (c) the demultiple result using the single channel matching filter; (d) the demultiple result using the normal multichannel matching filter; (e) the demultiple result using the pseudomultichannel matching filter; and (f) the demultiple result using the expanded multichannel matching filter. The expanded multichannel matching filter compared to the others produces the superior demultiple result which preserves the continuity of the primary event. 
between Figure 4 and Figure 5a, evidences a small amount of primary energy leakage.

The common-offset-domain MEMUL result is shown in Figure 6. Comparing Figures 5 and 6, we can draw the following conclusions:

1) A CMP-domain MEMUL can remove flat multiple reflections effectively. In order to attenuate the steeply dipping events, such as the diffraction multiples, we need to perform MEMUL in the common-offset domain.
2) The common-offset-domain MEMUL tends to degrade the primary energy as well. The CMP-domain implementation preserves the primary energy better.

A cascaded application of MEMUL has proven to be effective in practice, because the simple surface-related multiple reverberations and the peg-leg multiples have different amplitude decays, and attempting to attenuate both at the same time would need a very aggressive adaption, which has susceptibility of degrading the primary. When attempting to attack

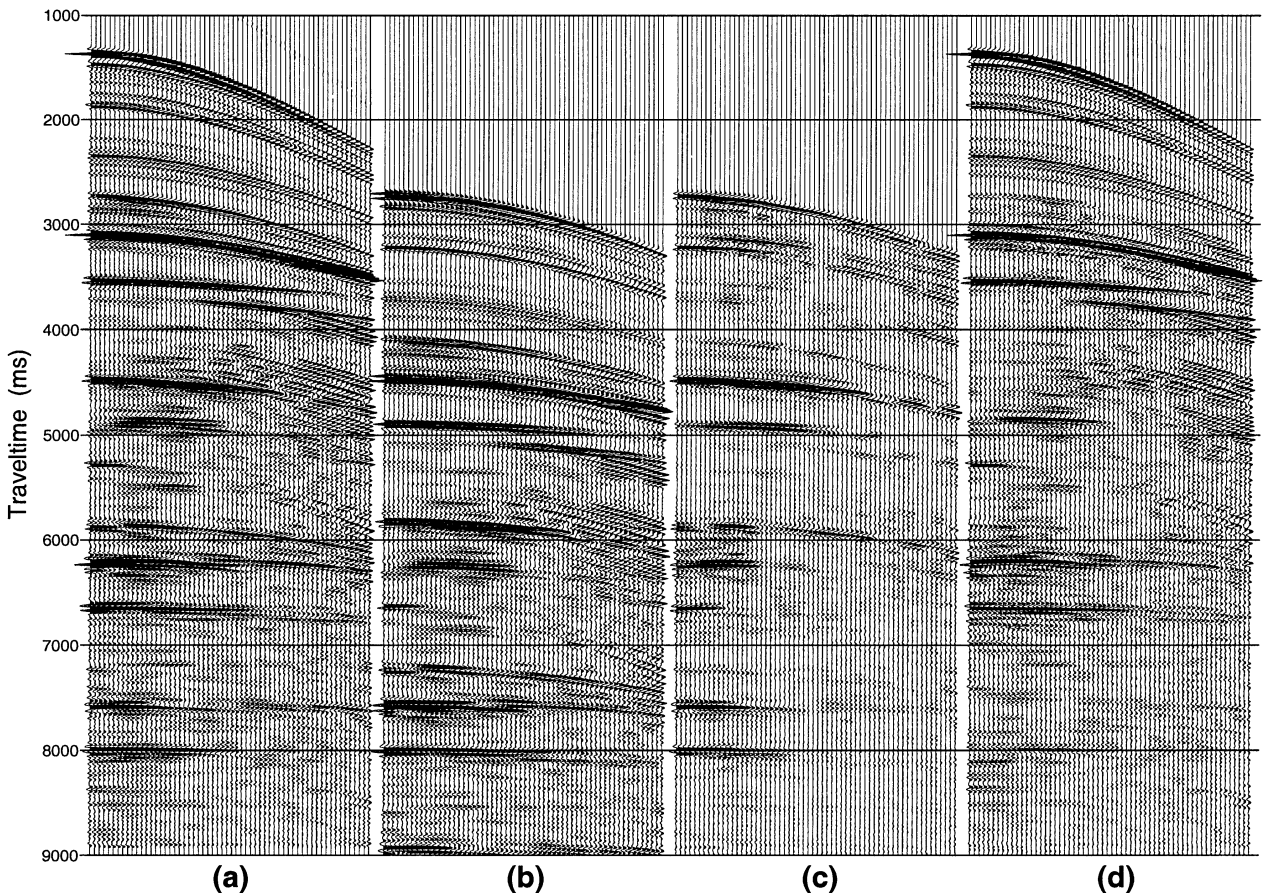

FIG. 3. Illustration of the moveout-equation-based multiple attenuation (MEMUL) method using the expanded subtraction: (a) a CMP gather with multiples, (b) the multiple model gather, (c) the multiple energy subtracted from the data gather, and (d) the final gather after multiple attenuation.

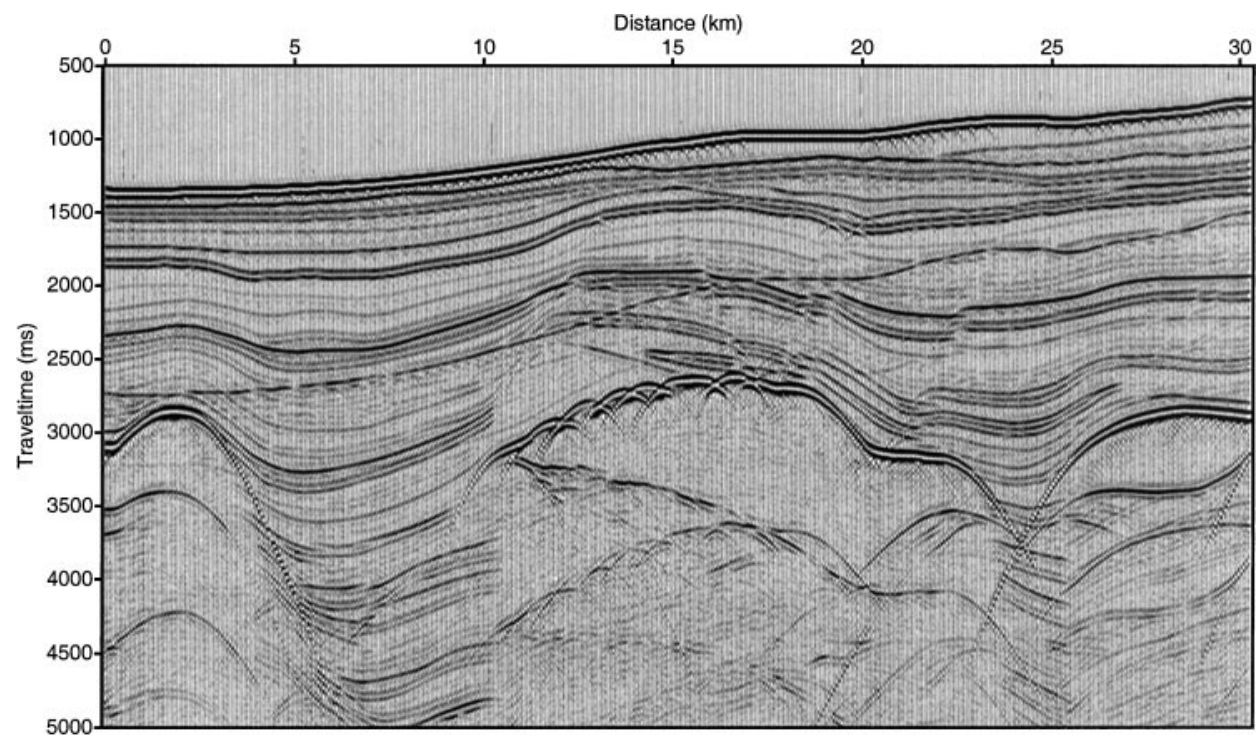

FIG. 4. The brute stack section, which shows clearly the multiples of water-bottom reflection and of the top and bottom reflections of three salt bodies. 
the simple water-layer reverberations, a constant water-layer velocity (instead of the rms velocity function) is used in multiple prediction, while use of the rms velocity function is for the prediction of water-layer peg-leg multiples. The cascade steps can be performed in the CMP gather and in the common-offset section alternately. However, one should be aware of that the common-offset domain MEMUL can attenuate primary energy, since the primary and multiple reflections has less moveout difference in this domain than in the CMP domain.

The Pluto 1.5 synthetic data set is two dimensional. In practice, if there are 3D effects in the water bottom, or if the water velocity is imperfect, there will be variations in the time shifts needed to match the model and data traces. The shifts will vary in time and space. Strong variation of time shifts in time and space will restrict the size of sliding widow in time and the number of channels used in multichannel matching. One should constrain the adaptive subtraction using a short matching filter, if possible.

\section{CONCLUSIONS}

While the efficiency and accuracy of multiple modeling are important to demultiple processing, the emphasis of this paper is on the expanded multichannel matched filtering in the multiple subtraction phase. The concept of combining the multichannel adaptive subtraction and cross-equalization into the expanded multichannel matching seems to be an appropriate implementation for constrained minimum-energybased multiple subtraction. The expanded multichannel matching filter expands the $2 \mathrm{D}$ operator in the time-space domain of a normal multichannel matching filter into a 3D operator in the time-space-space domain. It makes the adaptive subtraction effective, and can be used in conjunction with an existing wave-equation-based multiple modeling method. The MEMUL method using moveout-equation-based multiple prediction deserves to be an option added to the long list of demultiple algorithms, as we expect that the effectiveness of any algorithm is really data dependent.
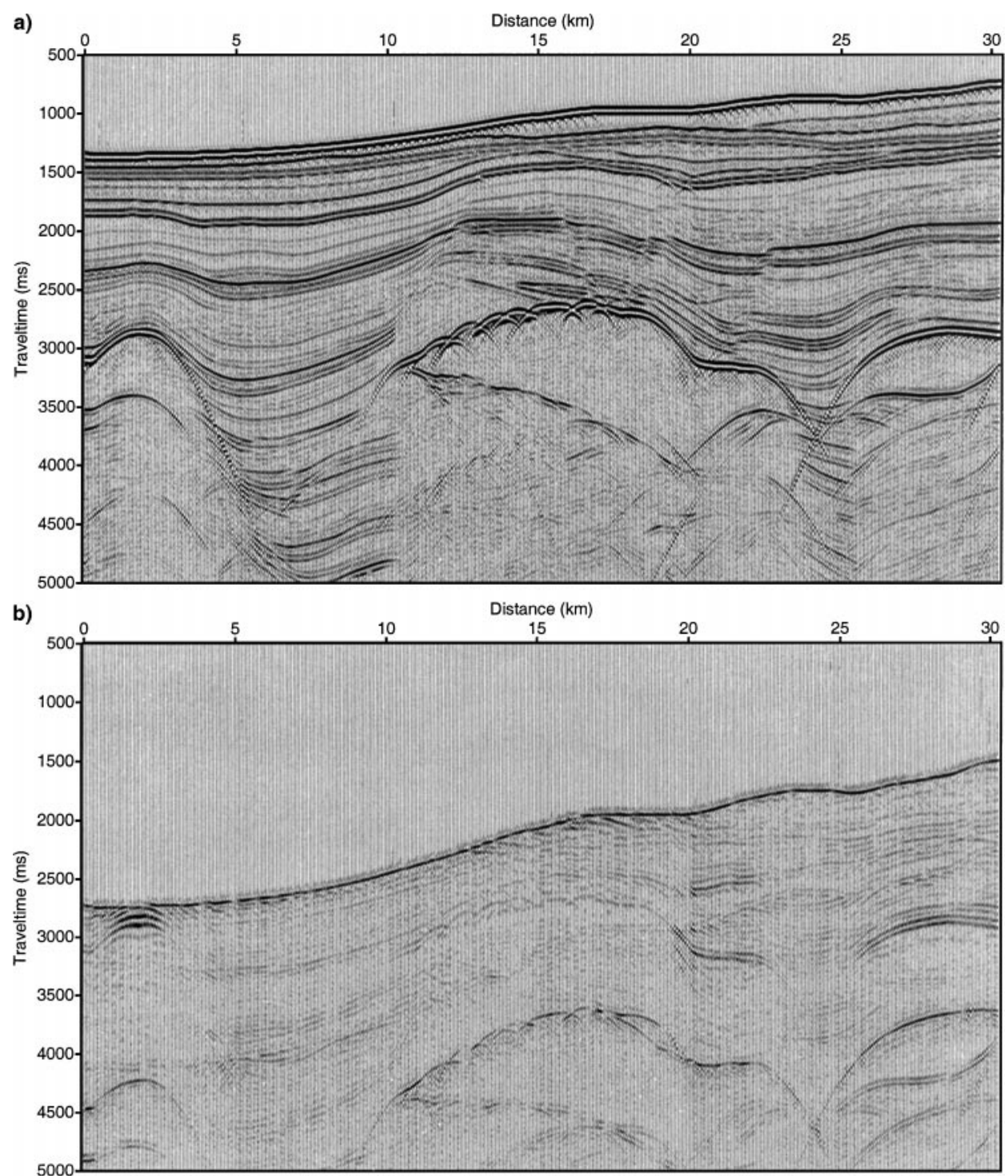

FIG. 5. MEMUL using the expanded multichannel matching filter in the CMP domain: (a) the stack section after multiple attenuation, and (b) the energy removed by the demultiple processing. 

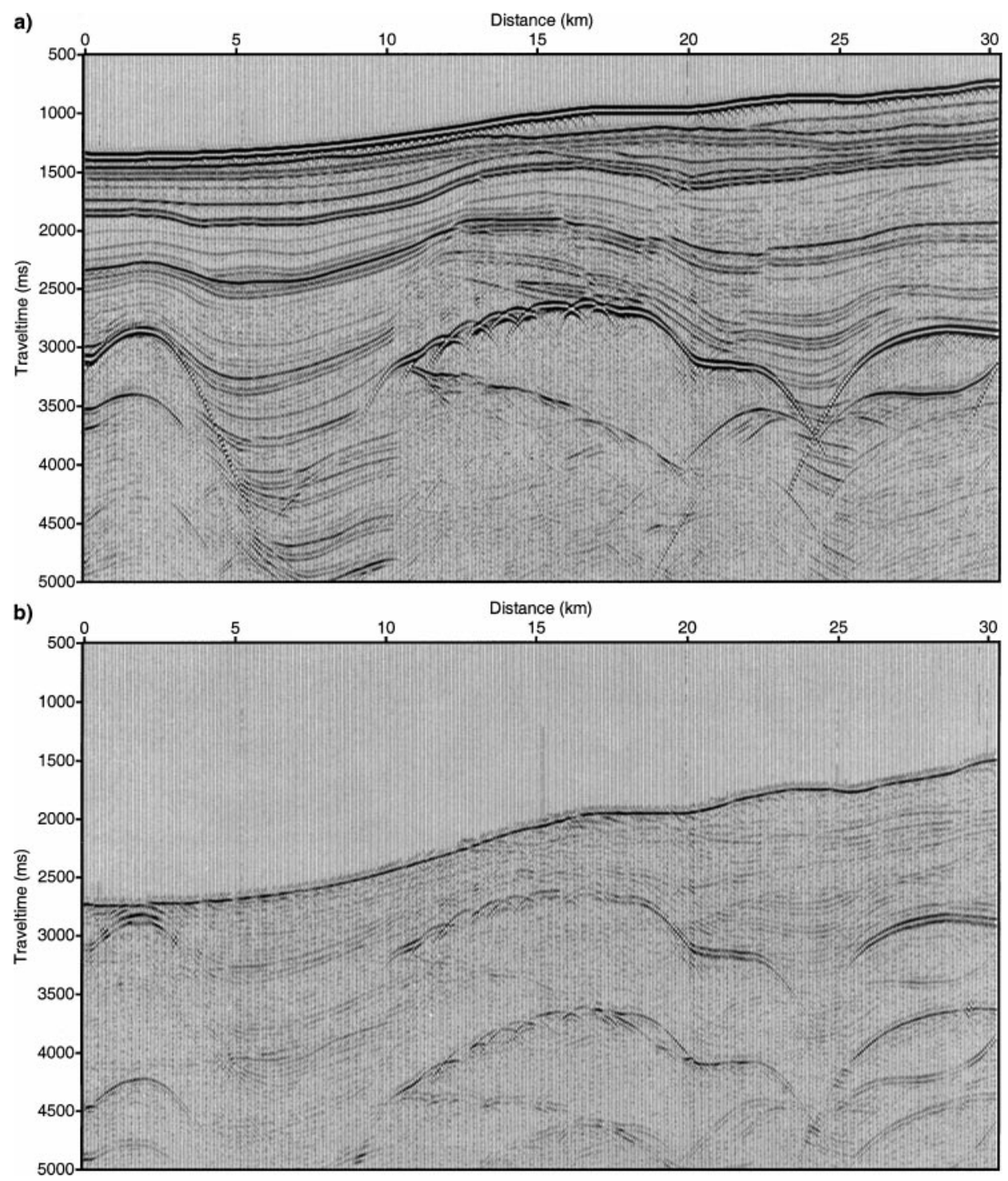

FIG. 6. MEMUL using the expanded multichannel matching filter in the common-offset domain: (a) the stack section after multiple attenuation, and (b) the energy removed by the demultiple processing.

\section{ACKNOWLEDGMENTS}

Stuart Merrylees conducted extensive experiment on multichannel matched filtering in conjunction with different multiple prediction methods. Eric Verschuur and an anonymous referee provided constructive comments concerning on the clarity of the presentation. The paper is published with permission of Robertson Research International.

\section{REFERENCES}

Berkhout, A. J., and Verschuur, D. J., 1997, Estimation of multiple scattering by iterative inversion, part I-Theoretical considerations: Geophysics, 62, 1586-1595.

Berryhill, J. R., and Kim, Y. C., 1986, Deep-water peg legs and multiples: Emulation and suppression: Geophysics, 51, 21772184

Bishop, K., Keliher, J., Paffenholz, J., Stoughton, D., Michell, S., Ergas, R., and Hadidi, M., 2001, Investigation of vendor demultiple technology for complex subsalt geology: 71st Ann. Internat. Mtg., Soc. Expl. Geophys., Expanded Abstracts, 12731276.
Monk, D. J., 1993, Wave-equation multiple suppression using constrained cross-equalization: Geophys. Prosp., 41, 725-736.

Nuttall, A. H., 1966, On the quadrature approximation to the Hilbert transform of modulated signals: IEEE Proc., 54, 1458 1459.

Stoughton, D., Stefani, J, and Michell, S., 2001, 2-D elastic model for wavefield investigations of subsalt objectives, deep water Gulf of Mexico: 71st Ann. Internat. Mtg., Soc. Expl. Geophys., Expanded Abstracts, 1269-1272.

Treitel, S., 1970, Principles of digital multichannel filtering: Geophysics, 35, 785-811.

Verschuur, D. J., and Berkhout, A. J., 1997, Estimation of multiple scattering by iterative inversion, part II-Practical aspects and examples: Geophysics, 62, 1596-1611.

Verschuur, D. J., Berkhout, A. J., and Wapenaar, C. P. A., 1992, Adaptive surface-related multiple elimination: Geophysics, 57, 11661177.

Wiggins, J. W., 1988, Attenuation of complex water-bottom multiples by wave-equation-based prediction and subtraction: Geophysics, $\mathbf{5 3}$ $1527-1539$.

Wiggins, R. A., and Robinson, E. A., 1965, Recursive solution of the multichannel filtering problem: J. Geophys. Res., 70, 1885-1891.

Zhou, B., 1993, Characterization and elimination of marine seismic reverberations: Ph.D. diss., The Flinders University of South Australia. 
APPENDIX A

\section{MOVEOUT-EQUATION-BASED MULTIPLE PREDICTION}

A multiple-model trace is predicted by shifting the sample in the original trace by the amount of time calculated using moveout equations, and flipping the polarity:

$$
m(t)=-y(t-\Delta t(t)),
$$

where $y(t)$ is the original data trace, and $m(t)$ is the multiple model trace. This transformed sample corresponds to the multiple event generated by the primary event making one additional traversal in the water layer.

For the multiple-model trace $m(t)$, given the sample time $t$, the zero-offset time $\tilde{t}_{0}(t)$ is estimated by

$$
\tilde{t}_{0}(t)=\sqrt{t^{2}-\frac{h^{2}}{v_{m}^{2}\left(\tilde{t}_{0}\right)}},
$$

where $h$ is the source-receiver offset, and $v_{m}$ is the moveout velocity of the multiples. Considering only first-order water reverberations, the zero-offset time of the original event should be

$$
t_{0}(t)=\tilde{t}_{0}(t)-t_{w},
$$

where $t_{w}$ is the vertical two-way time of the water layer, and $t_{0}$ with or without the tilde differentiate the zero-offset times of the multiple model gather and the original data gather. The corresponding arrival time of the primary reflection is then given by

$$
t_{p}\left(t_{0}\right)=\sqrt{t_{0}^{2}+\frac{h^{2}}{v_{r m s}^{2}\left(t_{0}\right)}},
$$

where $v_{r m s}\left(t_{0}\right)$ is the rms velocity. Note that $t_{p}\left(t_{0} \equiv \tilde{t}_{0}(t)-t_{w}\right)=$ $t_{p}(t)$, such that the time shift in transform equation (A-1) can be calculated by

$$
\Delta t(t)=t-t_{p}(t)
$$

Equivalently, the transform equation is represented as

$$
m(t)=-y\left(t_{p}(t)\right) .
$$

This operation maps the primary event of the original gather into the first-order multiple. Given the rms velocity $v_{\text {rms }}\left(t_{0}\right)$, the multiple moveout velocity $v_{m}\left(\tilde{t}_{0}\right)$ in equation (A-2) is defined as

$$
v_{m}\left(\tilde{t}_{0} \equiv t_{0}+t_{w}\right)=\sqrt{\frac{t_{0} v_{r m s}^{2}\left(t_{0}\right)+t_{w} v_{w}^{2}}{t_{0}+t_{w}}},
$$

where $v_{w}$ is the water-layer velocity.

For the high-order multiples, I define the multiple moveout velocity as

$$
v_{m}\left(\tilde{t}_{0} \equiv t_{0}+k t_{w}\right)=\sqrt{\frac{t_{0} v_{r m s}^{2}\left(t_{0}\right)+k t_{w} v_{w}^{2}}{t_{0}+k t_{w}}},
$$

where $k$ is the order of the water-layer multiple. The relation of the zero-offset times between the original data trace and multiple-model trace is

$$
t_{0}(t)=\tilde{t}_{0}(t)-k t_{w}
$$

This high-order option is useful in practice when one attempts to model a specified multiple reflection in a cascaded manner.

The moveout-equation-based multiple prediction method may be fast and effective enough to be used when the time or finances are limited, which is often the case on large modern 3D surveys. Its disadvantage is that the multiple modeling is limited to approximately $1 \mathrm{D}$ subsurface, though it may be extended to two dimensions in the time and the offset.

\section{APPENDIX B \\ DERIVATION OF THE EXPANDED MULTICHANNEL MATCHING FILTER}

If a multiple-model trace is defined as

$$
m(t)=\int_{0}^{+\infty} A(\omega) \cos [\omega t+\theta(\omega)] d \omega,
$$

its Hilbert transform, using the narrowband approximation (Nuttall, 1966), changes the cosine to the sine:

$$
m^{H}(t)=\int_{0}^{+\infty} A(\omega) \sin [\omega t+\theta(\omega)] d \omega,
$$

where $A(\omega)$ and $\theta(\omega)$ are the amplitude and phase spectra. Their derivatives with respect to the time $t$ are

$$
\dot{m}(t)=\int_{0}^{+\infty} A(\omega) \cos ^{\prime}[\omega t+\theta(\omega)] d \omega
$$

and

$$
\dot{m}^{H}(t)=\int_{0}^{+\infty} A(\omega) \sin ^{\prime}[\omega t+\theta(\omega)] d \omega,
$$

respectively. These four traces $[m(t)$ and three traces originated from $m(t)]$ will be used to define the following expanded multi- channel matching filter.

Given the model trace $m(t)$, the analytic trace is represented as

$$
M(t)=m(t)+i m^{H}(t)=\int_{0}^{+\infty} A(\omega) e^{i[\omega t+\theta(\omega)]} d \omega .
$$

For a data trace $y(t)$, we may also define the associated analytic trace as

$$
Y(t)=\int_{0}^{+\infty} \alpha(\omega) e^{i[\omega(t-\tau(t))+\theta(\omega)-\phi(\omega)]} d \omega,
$$

where $\alpha(\omega)$ is the amplitude, differing from $A(\omega), \tau(t)$ is the time shift, and $\phi(\omega)$ is the phase difference. The real part of $Y(t)$ is the data trace $y(t)$,

$$
\begin{aligned}
y(t)= & \int_{0}^{+\infty} \alpha(\omega)\{\cos [\omega(t-\tau(t))+\theta(\omega)] \cos [\phi(\omega)] \\
& +\sin [\omega(t-\tau(t))+\theta(\omega)] \sin [\phi(\omega)]\} d \omega . \quad(\mathrm{B}-7)
\end{aligned}
$$


Assuming that the time shift $\tau(t)$ is small, we can use the following approximations:

$$
\begin{aligned}
\cos [\omega(t & -\tau(t))+\theta(\omega)] \\
& \approx \cos [\omega t+\theta(\omega)]-\tau(t) \cos ^{\prime}[\omega t+\theta(\omega)]
\end{aligned}
$$

and

$$
\begin{aligned}
\sin [\omega(t & -\tau(t))+\theta(\omega)] \\
& \approx \sin [\omega t+\theta(\omega)]-\tau(t) \sin ^{\prime}[\omega t+\theta(\omega)] .
\end{aligned}
$$

Substituting them into equation (B-7) and making some rearrangements,

$$
\begin{aligned}
y(t) \approx & \int_{0}^{+\infty}\left[A(\omega) \cos [\omega t+\theta(\omega)] \frac{\alpha(\omega) \cos [\phi(\omega)]}{A(\omega)}\right. \\
& -A(\omega) \cos ^{\prime}[\omega t+\theta(\omega)] \frac{\alpha(\omega) \tau(t) \cos [\phi(\omega)]}{A(\omega)} \\
& +A(\omega) \sin [\omega t+\theta(\omega)] \frac{\alpha(\omega) \sin [\phi(\omega)]}{A(\omega)} \\
& \left.-A(\omega) \sin ^{\prime}[\omega t+\theta(\omega)] \frac{\alpha(\omega) \tau(t) \sin [\phi(\omega)]}{A(\omega)}\right] d \omega,
\end{aligned}
$$

we have the following multichannel convolution expression:

$$
\begin{aligned}
y(t)= & f_{1}(t) * m(t)+f_{2}(t) * \dot{m}(t)+f_{3}(t) * m^{H}(t) \\
& +f_{4}(t) * \dot{m}^{H}(t),
\end{aligned}
$$

where $f_{i}$ are the convolution operators. This convolution formula is used to define the pseudomultichannel matching filter [equation (5)] and the expanded multichannel matching filter [equation (6)].

The derivation in this Appendix follows closely Monk's (1993) constrained cross-equalization in which all of parameters, $\alpha / A, \phi$, and $\tau$, were assumed to be frequency- and timeindependent constants, and all of the convolution coefficients in equation (B-9) became scale-weighting coefficients. In contrast, the multichannel convolution formula (B-9) recognizes that those prementioned parameters are frequency and time dependent, and therefore defines the time-variant convolution operators.

To balance the sensitivities of four convolution operators $f_{i}(t)$ in equation (B-9), two time-derivative traces $\dot{m}(t)$ and $\dot{m}^{H}(t)$ are equalized so as to have same magnitude as the original trace $m(t)$ and its Hilbert transform $m^{H}(t)$. 